\title{
The balanced optimization of the project quality schedule and cost based on Genetic Algorithm
}

\author{
Xuemei Du, a ${ }^{1,}$, Xing Tang ${ }^{1, b}$, Hui Gao ${ }^{1, c}$ and Zongqiu Zhou ${ }^{1, d}$ \\ ${ }^{1}$ School of Economics and Management, Tongji University, Shanghai, China

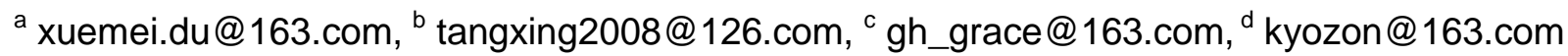

\begin{abstract}
Keywords: Cost of quality; Multi-objective optimization; Quality quantification; Genetic Algorithm Abstract. The multi-objective management in engineering projects is to reduce cost, improve quality and shorten the cycle. The optimization of these three objects is very significant to improve the comprehensive benefit of projects. In this paper, project quality is quantified by the cost of quality (COQ), and a mathematic model of quality-schedule-cost multi-objective balanced optimization is proposed, which can be solved by Genetic Algorithm (GA). Considering different construction requirements in engineering practice, three objectives are given weights to make decision-making more coherent and responsive in project management. Finally, applicability and efficiency of this method are verified by an example study.
\end{abstract}

\section{Introduction}

Project management (PM) is to maintain the quality within limited time and approved budget, including all aspects of planning, organization, control and coordination throughout the project lifecycle. Quality, schedule and cost management are intimately interrelated which are all aiming at the maximum profit of the project, and to control one of them will inevitably affect the other two. So it is necessary to find an optimal trade-off among the three objectives. However, the role and importance of quality are usually neglected by managers, who are lack of effective methods to quantify quality objective. As such, we will use COQ to quantify the quality objective, and further establish quality, schedule and cost multi-objective optimization model and select an appropriate construction mode to maximize revenue for different types of projects.

\section{Literature Review}

Recently multi-objective optimization management is a hot spot in PM, which can achieve an optimum trade-off and improve the efficiency and effectiveness of the project implementation. The initial research is limited to single or $\mathrm{Bi}$ objective optimization, while the multi-objective optimization is ignored. F.S. Pan (2010) makes the research for optimal scheduling based on particle swarm optimization [1]. Considering the fuzziness with uncertain information, C.S. Yi (2015) proposes a fuzzy expected value model for the time-cost-quality trade-off with multiple modes according to the credibility theory [2]. S. H. Razavi (2013) combines the fuzzy goal and grey linear programming to solve trade-off optimization, which can help managers determine the best combination of cost, time, and quality of the activities [3].

The difficulty in the process of multi-objective optimization is to quantify the quality. Robert McKim (2000) defines quality as "Compliance with contract specifications "[4]; G.M. Cui (2006) uses the repair rate of sub-unit project for quality of construction [5]; Corbet (1997) creates a quality index according to the engineering experts' evaluation on 10 quality attributes that define quality[6]. Y.B. Cao (2009) introduces the value engineering to the quality evaluation and uses the improved immune genetic algorithm to solve the problem [7]. Referring to the methods of foreign project management, Yongkun Wang (2005) integrates the multi-objective management through earned value theory and puts forward the algorithm and measurement of the earned value in quality [8].

In fact, although many traditional methods can achieve good results, but the better solution is obtained when GA is applied for the problem due to its current effectiveness, strong robustness, and simple implementation. In addition, the aforesaid literatures mainly adopt subjective quality evaluation methods so that results are not very scientific and accurate, since it is difficult to quantify 
quality into objective value which is related to cost and schedule. COQ is measurable and can make quality evaluation easy. So the multi-objective optimization based on COQ with GA has certain theoretical and practical significance.

\section{Modeling process}

Quantification of project quality. The cost of quality was put forward by AV Feigenbaum in early 1950s. The cost associated with quality is divided into two categories: cost of good quality and cost of poor quality. [9]Prevention cost $\left(Q_{p c}\right)$ and appraisal cost $\left(Q_{a c}\right)$ are associated with good quality, while failure cost results from poor quality. Failure cost includes internal failure cost $\left(Q_{i c}\right)$ and external failure Cost $\left(Q_{e c}\right)$.

The object of this research is Sub project. Quality cost items in construction enterprise are set up according to the literature [9] and construction practice. The Sub projects share the total prevention cost of construction enterprise. The proportion of prevention cost $\left(V_{p c}\right)$ can be expressed as Eq. 1.

$$
V_{p c}=\frac{Q_{t}}{\sum_{i=1}^{n} p(i)} .
$$

$Q_{t}$ is the total prevention cost for the sub project; $\sum_{i=1}^{n} p(i)$ is the sum contract price of all the completed sub projects during the construction period.

The appraisal cost and failure cost of the proposed sub project are collected from other similar projects. So is the contract price which is expressed by $P$. The ratio of COQ $\left(V_{q}\right)$ and COQ $(Q)$ are calculated:

$$
\begin{aligned}
& V_{q}=\frac{\sum_{i=1}^{n}\left(Q_{a c}(i)+Q_{i c}(i)+Q_{e c}(i)\right)}{\sum_{i=1}^{n} p(i)}+V_{p c}, \\
& Q=P \times V_{q} .
\end{aligned}
$$

Objective functions. If sub project can be completed ahead of schedule with good quality, construction side will be awarded $P_{a}$ yuan per day; If not completed within schedule $\left(T_{p}\right)$, contractor needs to bear the fines of $P_{f}$ yuan per day. As quality objective can be converted into computable values, the minimum expenditure function is obtained in Eq.3. $F(j)$ is the project expenditure for construction model $j(j=1,2, \mathrm{~K}$ Nind $)$.

$$
F=\min F(j) \text {. }
$$

Project schedule: The economic indicator of schedule is indirect cost such as management fees, which is proportional to the duration of the sub project $\left(T_{c}(j)\right)$. A is a collection of sub projects on a critical path in the project through network planning technology.

$$
T_{c}(j)=\sum_{i \in A} T_{i}(j) \text {. }
$$

Cost and quality cost: the cost refers to the measures fee and the sum of the direct cost of materials, labor and machinery (excluding management fees). Cost and COQ of the project summary from all the sub projects as follows:

$$
\begin{aligned}
& C_{z}(j)=\sum_{i=1}^{n} C_{z(i)}(j), \\
& Q(j)=\sum_{i=1}^{n} Q_{(i)}(j) .
\end{aligned}
$$

$\sum_{i=1}^{n} C_{z(i)}(j)$ and $\sum_{i=1}^{n} Q_{(i)}(j)$ are the cost and COQ of all sub projects under the construction mode $j$ respectively.

In practice, different kinds of have different focus on quality, cost or schedule. The weight value can be given to make the model more flexible and applicable. The trade-off optimization can be taken 
as a special case with same weight. $F(j)$ is not a project expenditure any more, but a evaluation function of the construction mode. $C_{j}$ is an indirect cost per day. $\lambda_{t}$ is schedule weight, $\lambda_{c}$ is cost weight, $\lambda_{q}$ is quality weight. The objective equation is as follows:

$$
\begin{aligned}
& F=\min F(j), \\
& \text { If } T_{c}>T_{p} F(j)=\lambda_{t} T_{c}(j) \cdot C_{j}+\lambda_{c} \cdot C_{z}(j)+\lambda_{q} \cdot Q(j)+\left(T_{c}(j)-T_{p}\right) \cdot P_{f}, \\
& \text { If } T_{c} \leq T_{p} F(j)=\lambda_{t} T_{c}(j) \cdot C_{j}+\lambda_{c} \cdot C_{z}(j)+\lambda_{q} \cdot Q(j)+\left(T_{p}-T_{c}(j)\right) \cdot P_{a}, \\
& T_{c}(j)=\sum_{i \in A} T_{i}(j), \\
& Q(j)=\sum_{i=1}^{n} Q_{(i)}(j), \\
& C_{z}(j)=\sum_{i=1}^{n} C_{z(i)}(j), \\
& \lambda_{t}+\lambda_{c}+\lambda_{q}=1 .
\end{aligned}
$$

Genetic algorithm. The genetic algorithm uses a binary encoding mode, and construction model is coded as chromosome. This paper adopts roulette wheel selection method, three-points crossover and uniform mutation referred to [10].

\section{Computation example}

Construction companies rarely conduct quality cost management, so the collection of data on COQ is very difficult. This paper uses a numerical example to solve the model through the MATLAB, and the example is adapted from the literature [11], as shown in Table 1. The duration, direct cost and failure cost of 7 sub projects varies with the change of the construction approach, and each sub project can adopt one of the three approaches.

Table 1 The parameters of each sub project in a numerical example

\begin{tabular}{ccccc}
\hline $\begin{array}{c}\text { Sub-project } \\
\text { (i) }\end{array}$ & $\begin{array}{c}\text { Successor } \\
\text { sub project }\end{array}$ & $\begin{array}{c}\text { Duration } \\
\text { [day] }\end{array}$ & Direct cost [yuan] & Failure cost [yuan] \\
\hline A & B,C,D & $(14,20,24)$ & $(230230,185270,160340)$ & $(10800,16650,20600)$ \\
B & E & $(6,8,12)$ & $(80510,65340,40290)$ & $(7000,5760,3850)$ \\
C & E & $(7,10,12)$ & $(50340,32570,20620)$ & $(4853,3624,2564)$ \\
D & F & $(14,16,20)$ & $(500240,485370,450680)$ & $(42153,36458,30427)$ \\
E & G & $(20,23,27)$ & $(210150,195240,170370)$ & $(20146,16657,13752)$ \\
F & G & $(14,18,24)$ & $(350670,328270,310370)$ & $(35245,29527,24853)$ \\
G & - & $(9,15,18)$ & $(280260,250340,208620)$ & $(28432,22452,19342)$
\end{tabular}

Other parameters are as follows: the population size (Nind=1000), the maximum evolutionary algebra $(\mathrm{N}=300)$, the crossover probability $\left(\mathrm{P}_{\mathrm{c}}=0.7\right)$, mutation probability $(\mathrm{Pm}=0.05)$, indirect cost (5000 yuan/day), $P_{a}=300 \mathrm{yuan} / \mathrm{day}, P_{f}=500 \mathrm{yuan} / \mathrm{day}$.

Project schedule: Through the network planning technique, construction network chart is showed in Fig.1. The possible scheduling sequences are ABEG, ACEG, ADFG. A is the time matrix of the three construction paths. The calculation steps of the duration $\left(T_{c}\right)$ are as follows:

$$
\begin{aligned}
A & =\left[\begin{array}{ccccccccccccccccccccc}
14 & 20 & 24 & 6 & 8 & 12 & 0 & 0 & 0 & 0 & 0 & 0 & 22 & 23 & 27 & 0 & 0 & 0 & 9 & 15 & 18 \\
14 & 20 & 24 & 0 & 0 & 0 & 7 & 10 & 12 & 0 & 0 & 0 & 22 & 23 & 27 & 0 & 0 & 0 & 9 & 15 & 18 \\
14 & 20 & 24 & 0 & 0 & 0 & 0 & 0 & 0 & 14 & 16 & 20 & 0 & 0 & 0 & 14 & 18 & 24 & 9 & 15 & 18
\end{array}\right]^{T} \\
B & =\operatorname{pop}^{*} A \\
C & =\max \left(B^{\prime}\right), \\
T_{c} & =C^{\prime} .
\end{aligned}
$$




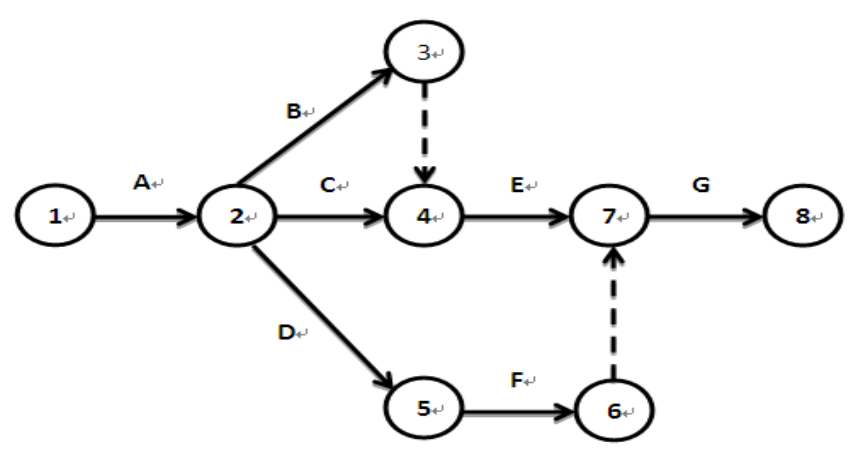

Fig.1. A project Network structure

Cost and quality cost: $C_{z z}$ is the cost matrix of the sub projects under three construction approaches; $Q_{s s}$ is the quality cost matrix of the sub projects under three construction approaches.

$$
\begin{aligned}
C_{z z}= & {[230230,185270,160340,80510,65340,40290,50340,32570,20620,500240,485370,} \\
& 450680,210150,195240,170370,350670,328270,310370,280260,250340,208620]^{\mathrm{T}} \\
Q_{s s}= & {[10800,16650,20600,7000,5760,3850,4853,3624,2564,42153,36458,} \\
& 30427,20146,16657,13752,35245,29527,24853,28432,22452,19342]^{\mathrm{T}} \\
C_{z}= & \text { pop } * C_{z z}, \\
Q= & \text { pop } * Q_{s s} .
\end{aligned}
$$

\begin{tabular}{|c|c|c|c|c|c|}
\hline No. & The optimum solutions & $\begin{array}{c}\text { Duration } \\
\text { [day] }\end{array}$ & $\begin{array}{l}\text { Direct cost } \\
\text { [yuan] }\end{array}$ & $\begin{array}{l}\text { Failure cost } \\
\text { [yuan] }\end{array}$ & Expenditure[yuan] \\
\hline 1 & $\{010001001001001010001\}$ & 77 & 1404120 & 116112 & 1908732 \\
\hline 2 & $\{1000001001001001010001\}$ & 71 & 1449080 & 110262 & 1914842 \\
\hline 3 & $\{010001010001001010001\}$ & 77 & 1416070 & 117172 & 1921742 \\
\hline 4 & 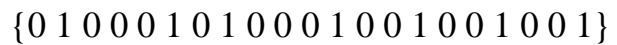 & 82 & 1398170 & 112498 & 1926668 \\
\hline
\end{tabular}

After the operation of the Matlab program, the optimal solution is obtained without considering the weight of the objectives, as shown in Table 2.

Table 2 The sample of the multi-objective trade-off optimization solutions

The weight of same objective is different in different projects. For instance, the project of city's landmark will prefer to ensure quality while emergency disaster relief project must ensure schedule. Due to space limitations, only the results of the priority to ensure quality are listed here. $\lambda_{t}=\lambda_{c}=0.1$, $\lambda_{q}=0.8$, and the other parameters is invariant. The solutions are showed in Table 3.

Table 3 The sample of the quality priority optimization solutions

\begin{tabular}{|c|c|c|c|c|c|c|}
\hline No. & The optimum solutions & $\begin{array}{c}\text { Duration } \\
\text { [day] }\end{array}$ & $\begin{array}{c}\text { Direct cost } \\
\text { [yuan] }\end{array}$ & $\begin{array}{c}\text { Failure } \\
\text { cost } \\
{[\text { yuan] }}\end{array}$ & Expenditure[yuan] & $\begin{array}{c}\text { F(j) } \\
\text { [yuan] }\end{array}$ \\
\hline 1 & $\{100001001001001001001\}$ & 76 & 1431180 & 105588 & 1919768 & 268590 \\
\hline 2 & $\{100001001001001010001\}$ & 71 & 1449080 & 110262 & 1914842 & 269120 \\
\hline 3 & $\{100001010001001001001\}$ & 76 & 1443130 & 106648 & 1932778 & 270630 \\
\hline 4 & $\{100001100001001001001\}$ & 76 & 1460900 & 107877 & 1951777 & 273390 \\
\hline
\end{tabular}


The weight value is arbitrary, and the purpose is to demonstrate the applicability of the model. It should be determined according to the historical data of similar projects and project characteristics. Mode 1 is the best solution to the project. By comparing these results in Table 3 and Table 2, we can find that the duration of project is stable with little change, and the cost increases while the quality cost is reduced. The table 3 shows greater expenditure in mode 1 than mode 2 but it's worth it as its' evaluation value $(F(j)$ )is the lowest. It proves that the method is valid to ensure the quality and reduce the quality cost under the selected construction mode, and can apply to other situations.

\section{Conclusions}

A new method to quantify quality of engineering project is put forward based on COQ. As quality cost can be compared, it deals with the difficulty in the evaluation caused by the different evaluation criteria of the various projects.

This paper advances a new model of schedule cost quality optimization, and verifies the feasibility of this model with an example, which has certain theoretical and practical significance. According to the priority of each objective, appropriate adjustment on weight can help managers select the appropriate construction mode effectively.

The data in the numerical example are not collected from the actual project, and may appear a little bias. In the future, we can also add resources, security, environment and other indicators in the model, and make multi-objective optimization model more comprehensive.

\section{References}

[1] F.S Pan et al: 2010 International Conference on E-Business and E-Government. (2015)

[2] C.S. Yi, J.B. Gao: Journal of Engineering Management in china. Vol. 29 (2015), p.129-133

[3] S.H. Razavi Hajiagha, H.A. Mahdiraji and S.S. Hashemi: The International Journal of Advanced Manufacturing Technology. Vol. 71 (2013), p. 117-126

[4] Robert McKim, TarekHegazy, Mohamed Attalla: Journal of Construction Engineering and Management, Vol.126 (2000), p. 137-141

[5] Q. Zang, G.M. Cui et al: Energy Conservation in china,Vol.9 (2006), p. 17-20

[6] Corbet, Deanna I: submitted to The Penn State University (1997)

[7] Y.B. Cao: submitted to Tianjin University (2009)

[8] Y.K. Wang, W.Q. Zhong: Journal of Liaoning Technical University,Vol.7 (2005), p. 639-641

[9] J.X. You: The application of quantitative analysis technology in enterprise quality cost management (Tongji University Press ,China 2005)

[10] E. Pérez, A. Lorenzana and M. Posada: submitted to Soft Computing (2015)

[11] S.X. Wang, X.L. Zhou: Journal OF Chongqing Jiaotong University in china, Vol.20 (2001), p.39-45 